\title{
Synchronized Power Saving Mechanism for WiMAX Networks
}

\author{
Aymen Belghith*, Amal Walha*, Bernard Cousin ${ }^{\dagger}$, Mohammad S. Obaidat ${ }^{\ddagger}$ \\ ${ }^{*}$ University of Sfax, Road of Aeroport Km 0.5, 3029 Sfax, Tunisia \\ aymen.belghith@gmail.com \\ † Université de Rennes I - IRISA, Campus de Beaulieu, 35042 Rennes Cedex, France \\ bernard.cousin@univ-rennes1.fr \\ $\ddagger$ Fordham University, 441 East Fordham Road, JMH 340, Bronx, New York 10458 \\ msobaidat@gmail.com
}

\begin{abstract}
Power conservation mechanisms allow the operator to meet the QoS requirements of its customers while offering mobility service for longer duration. Therefore, it is essential to maximize the battery life of WiMAX equipments. In this paper, we study the mechanisms of power conservation already proposed for the WiMAX network. We then propose an improved mechanism for energy conservation in the IEEE802.16e standard, taking into account the characteristics of the WiMAX network. Finally, we evaluate the various mechanisms of energy conservation in terms of energy consumption, mean delivery time and management throughput. We show through extensive simulations that our proposed mechanism retains more energy while providing a better mean delivery time and decreasing the management throughput.
\end{abstract}

\section{INTRODUCTION}

Today, energy costs, for several service providers, are important and constitute a large part of their operating expenses in case of mobile networks. Users require the support of mobility for all services and this is the role of the network access providers. Worldwide Interoperability for Microwave Access (WiMAX) is a wireless broadband technology providing a high throughput and a large coverage. WiMAX is essentially a new shortcut to name the IEEE 802.16 standard [1].

IEEE 802.16e [2], called mobile WiMAX, has been proposed to combine the mobility and broadband wireless communications to support mobile services based on the convergence of devices. This technology adds mobility to the IEEE 802.16d (Wireless MAN) standard and overcomes the short coverage of wireless local area network and Internet over cell phones. However, since end nodes become mobile subscriber stations (MSSs), they must reduce their energy consumption. Therefore, WiMAX devices can increase their battery life and prolong their operations [3]. A main idea of a power conservation mechanism consists of powering down the wireless interface to a lower state (the Sleep state) when deemed reasonable [4]. Other interesting types of techniques can be used to save energy such as the prediction of the Packet Interval Time (PIT) [5] and the choice of the QoS requirements to satisfy [6].

IEEE 802.16e defined an algorithm called Power Saving Mechanism (PSM) [7]. The PSM mechanism allows MSSs to save energy by introducing a new Sleep mode. The Sleep mode duration is between a minimum and a maximum sleep interval called Tmin and Tmax, respectively. Many research as in [8], [9], and [10] show that PSM can enhance system performance in term of power saving gain. The main problem of PSM that it does not take into account the mean delivery time as longer sleep cycle usually produces higher response time [11]. The mean delivery time represents the difference between the instant of the reception of data and the instant of its generation [12].

In [13], authors proposed a power saving algorithm that reduces the next sleep window interval to the mean between sleep intervals Tmin and Tmax. Consequently, the mean delivery time can be reduced. However, the total energy consumption increases. In [14], K. Min-Gon et al. proposed a power saving mechanism that considers the request period of each awakening event. This mechanisms adjusts PSM parameters, for example Tmin and Tmax, in order to minimize the energy consumption and mean delivery time. In [15], K. MinGon et al. slightly modified their power saving mechanism already proposed in [14]. The proposed algorithm is called Enhanced PSM (EPSM). Authors adjust PSM parameters taking into account the battery state of the mobile stations

In [5], authors proposed a Hidden Markov Model (HMM) that predicts the Packet Interval Time to reduce the energy consumed. They do not consider other parameters as the packet loss and the packet size because these parameters have a little impact on the energy consumption. Simulations results show that the mechanism proposed can reduce the energy consumption by $5 \%$ compared to PSM.

In [6], Jia Liu and al. proposed a mechanism that helps users select the suitable QoS requirements. These QoS requirements are selected once an optimal dual-threshold (on energy and delay) is determined using two dimensional Markov Chain model. In [16], in addition to the QoS requirements selection, authors proposed to make adjustments to the traffic. The adjustment decision depends on the arrival rate. Simulations results show promising performance compared to the Poisson arriving assumptions.

For the best of our knowledge, there is not yet any published power saving mechanism taking into account the technical characteristics of WiMAX. Examples of WiMAX technical 
characteristics are the structure of WiMAX frame as well as the radio resources and the management messages. The main management messages used in WiMAX are Downlink map (DL-MAP) and uplink map (UL-MAP). DL-MAP and ULMAP indicate the location of the downlink and uplink data bursts, respectively.

Two duplexing modes are supported in WiMAX: Time Division Duplexing (TDD) and Frequency Division Duplexing (FDD) [17]. The TDD mode uses the same frequency in the downlink (DL) direction (from the Base Station to the MSS) and in the uplink (UL) direction (from the MSS to the Base Station). Two different frequencies are used in the FDD duplexing mode. In this work, we are interested in the TDD mode. The Orthogonal Frequency Division Multiplexing (OFDM) physical layer in WiMAX considers the OFDM symbol as the unit of resource allocation. An OFDM symbol $\left(T_{s}\right)$ is computed as follows [18]:

$$
T_{s}=\frac{N_{F T T}}{n \times B W} \times(1+G)
$$

where $N_{F T T}$ represents the total number of subcarriers, $n$ represents the sampling factor, $B W$ represents the channel bandwidth, and $G$ represents the ratio of Cyclic Prefix $(C P)$ time to useful time.

In order to obtain an efficient energy consumption mechanism that takes into account WiMAX characteristics and can be really implemented in WiMAX devices, we propose, in this paper, a novel power saving algorithm, called Synchronized Power Saving Mechanism (SPSM), that reduces the energy consumption without suffering from high delivery time and management throughput.

This paper is organized as follows. In Section II, we describe some energy conservation mechanisms already proposed for WiMAX. Our enhancement will be detailed in Section III. We evaluate these power saving algorithms in Section IV in terms of total energy consumed, management messages throughput and mean delivery time. Finally we conclude our work in Section V.

\section{Existing EnERgy CONSERVATION MECHANisms}

In this section, we present the mechanism defined in the IEEE802.16e standard called PSM [2] as well as the wellknown existing mechanisms: Adaptive Sleep Mode Algorithm (ASMA) [13] and Adaptive PSM (APSM) [14]. We do not consider EPSM [15] and HMM-based predictive PSM [5] as they provides similar performance to APSM and PSM, respectively. Moreover, we do not evaluate mechanisms defined in [6] and [16] because we suppose that WiMAX users have defined QoS requirements and have already selected their WiMAX QoS classes. So, they do not modify their QoS requirements.

\section{A. Power Saving Mode in IEEE 802.16e}

An MSS can be in Awake or Sleep modes. It can receive and send bursts only in the Awake mode. When an MSS is in the Awake mode and does not have any data to send or to receive, it transmits a message called Mobility Sleep
Request (MOB-SLP-REQ) in order to request switching to the Sleep mode. The mobile subscriber station can switch to the economic energy (Sleep) mode only if it receives from the Base Station (BS) a message called Mobility Sleep Response (MOB-SLP-RSP) containing a positive indication. Note that the BS can send this response message without solicitation (without receiving a request) if an MSS remains for a long duration neither sending nor receiving bursts.

The Sleep mode uses two parameters: Tsleep and $L$. Tsleep represents the sleep window while $L$ represents the listening window. During Tsleep, the MSS transceiver is powered off and therefore it does not consume energy. However, during $L$, the MSS turns on its transceiver in order to verify if it has data to receive from the BS. The BS uses the MOB_TRF_IND message for downlink data delivery information. The duration of Tsleep is initially equal to Tmin. It is doubled when there is no data to send or receive during a sleep cycle without exceeding T max.

When an MSS receives a MOB_TRF_IND message with positive indication, it has to return in the Awake mode and Tsleep comes back equal to Tmin in the next sleep cycle. Otherwise, if the indication of the MOB_TRF_IND message is negative, the MSS remains in the Sleep mode while updating Tsleep.

\section{B. Adaptive Sleep Mode Algorithm}

In addition to the three common parameters of PSM that consist of Tmin, Tmax and L, ASMA adds two other parameters called $A V G$ and $A V G \max$ in order to overcome the shortcomings in the basic Sleep mode. $A V G$ is the sleep window that Tsleep takes after reaching maximum sleep window Tmax. It is computed as follows:

$$
A V G=\frac{T \min +T \max }{2}
$$

$A V G \max$ indicates the maximum interval of the sleep window once AVG is reached. Then, when there is still no data to send or receive, the sleep window alternates between $A V G$ and $A V G \max$ (see Fig. 1). Therefore, ASMA enhances the delivery time when Tsleep reaches high values. In fact, an MSS waits $A V G$ instead of $T \max$ before moving to the listening window. Note that this power saving algorithm reduces the delivery time only in low traffic load but raises the total energy wasted.

\section{Adaptive Power Saving Mechanism}

The basic idea of APSM is to update common parameters Tmin and Tmax depending on the value of the current sleep window called $T f$ (see Fig. 2). APSM adds two additional parameters, called $T M I N$ and $T M A X$, to the three common parameters of PSM. TMIN and TMAX represent the smallest and largest values of Tmin and Tmax, respectively.

Tmin and Tmax are updated basing on the following policies: (1) If $T f$ is equal to $T M I N$ and Tmin is smaller than $T \max$, then Tmax is divided by 2. (2) Or, if $T f$ is equal to Tmax and Tmax is smaller than TMAX,Tmax is regarded 


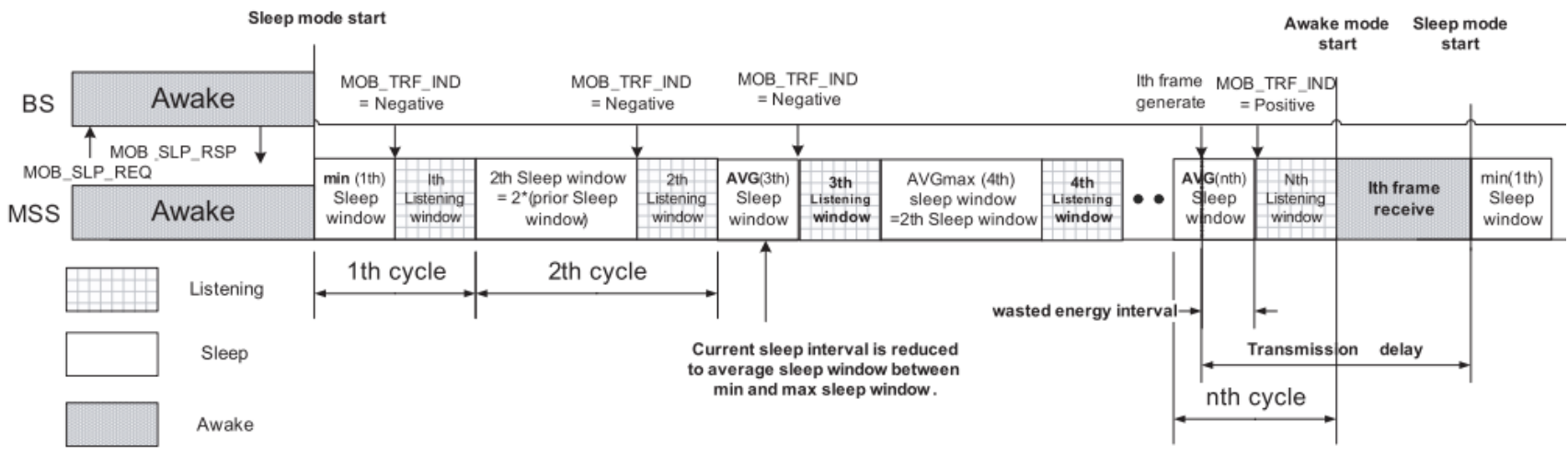

Fig. 1. Description of Adaptive Sleep Mode Algorithm (ASMA).

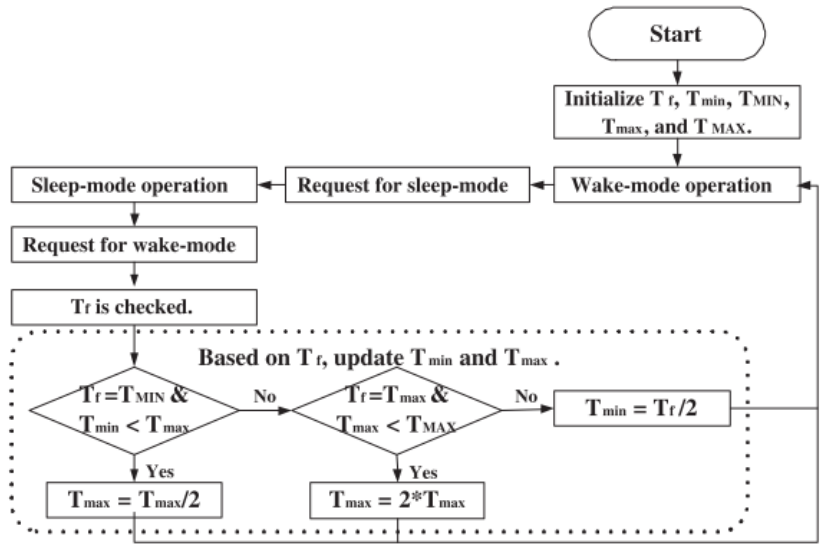

Fig. 2. Description of Adaptive Power Saving Mechanism (APSM).

as a relatively smaller size compared to the current sleep window. Therefore, Tmax doubles. (3) Otherwise, Tmin is updated to the half of $T f$.

These instructions are useful in order to take into account the traffic conditions. Note that if the values of Tmin and Tmax are too small, response delay may be lower but more energy consumption is expected. This can be explained by the fact that smaller Tmin and Tmax can induce more sleep cycles. However, if these parameters are set too large, higher energy conservation can be achieved but more response delay is expected.

\section{Synchronized Power SAVING Mechanism}

In this paper, we propose a novel mechanism of energy conservation that takes into account the characteristics of the WiMAX network. It helps MSSs mitigate the energy consumed and delivery time while reducing management messages exchanged. We firstly describe why a synchronization of the listening window with the DL-MAP and UL-MAP messages is important to provide improvements in the quality of service (QoS). Then we describe the configuration of our proposed power saving algorithm parameters.

\section{A. Synchronization of the listening window with the DL-MAP and UL-MAP messages}

The common idea of each power saving algorithm is that an MSS has to wake up in the listening window $(L)$ in order to verify if it has data to receive. Recall that the existing algorithms use MOB_TRF_IND messages for informing the MSSs that have downlink (DL) data to receive. Therefore, these messages make the MSSs switch to the Awake mode. However, any MSS needs an authorization to receive or send data by the DL-MAP and UL-MAP management messages, respectively. Consequently, we propose to add synchronization in order to take into account the frame structure and hence improve power saving performance.

When there is a downlink burst (resp. an uplink burst) generated before the DL-MAP (resp. UL-MAP) message, the BS can deliver this burst in the current WiMAX frame as it can inform the related MSS under the following conditions. The MSS has to be in the Awake mode or in the Sleep mode but in the listening window (and not in the sleep window). Evidently, the burst delivery can be postponed when there is no sufficient available radio resources. Figure 3 shows that the synchronization of the listening window with the DL-MAP and UL-MAP messages is essential to speed up the delivery time and hence improve the energy consumption. For the best of our knowledge, all existing power saving algorithms do not synchronize the sleep and listening windows with the DLMAP and UL-MAP messages.

Otherwise, if a downlink burst (resp. an uplink burst) is generated after the DL-MAP (resp. UL-MAP) message, the MSS misses the authorization to send in the current WiMAX frame. So this MSS has to wait for the next WiMAX frame for burst delivery. Figure 4 shows that in this case, the listening and sleep windows synchronization does not reduce the delivery time. Nonetheless, the synchronization is essential to improve the energy consumption as it is useless to wait the burst delivery in the current WiMAX frame.

Now, we show how we can eliminate additional management messages when taking into account the characteristics of WiMAX network. Since DL-MAP and UL-MAP messages are sent in each WiMAX frame, we propose to use them to 


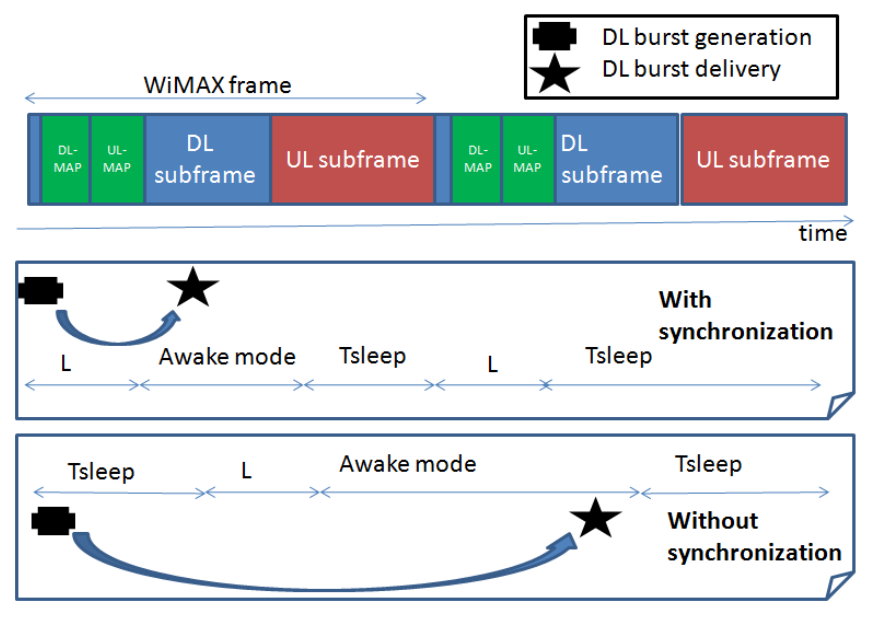

Fig. 3. DL burst delivery when generated before DL-MAP.

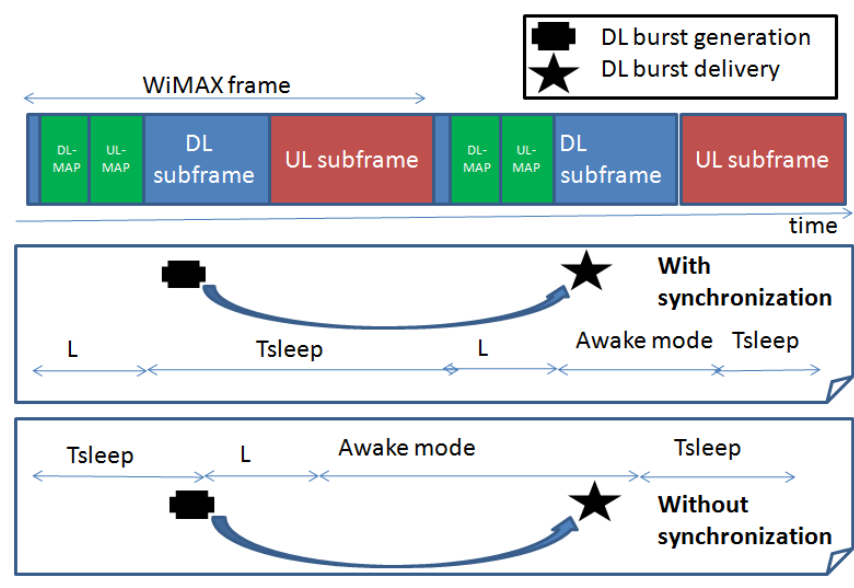

Fig. 4. DL burst delivery when generated after DL-MAP.

inform MSSs if they have to wake up. Recall that an MSS has to wake up when there are bursts to receive or sent in the following WiMAX frame. Evidently, the MSS has to be able to receive those management messages and therefore be in the Awake mode or in the listening window of the Sleep mode. This eliminates sending management message MOB_TRF_IND, which reduces energy consumption and the number of management messages sent.

\section{B. Parameters configuration}

Let us configure the different parameters which are used by any power saving algorithm: L, Tmin, Tmax, and Tsleep. Our algorithm does not need additional parameters to offer good performance. In addition of the DL-MAP and ULMAP messages, we note that the preamble and the Frame Control Header $(\mathrm{FCH})$ are important. The preamble is used for synchronization between the BS and the MSSs. Therefore, all users are already synchronized with the BS in order to be able to read the messages, independently of the power saving mechanism used. However, only our proposed power saving mechanism adds the synchronization of PSM parameters to enhance the system performance. The FCH, that immediately follows the preamble, contains the location and burst profile of the first downlink burst. Therefore, we consider that preamble and FCH are located in the listening window to enable the MSSs to receive them. Note that the IEEE 802.16 standard defined constant durations for the preamble and the $\mathrm{FCH}$. They are two and one OFDM symbols long, respectively [19]. OFDM symbol duration $T_{s}$ is computed in (1). Subsequently, we propose that the listening period $(\mathrm{L})$ is equal to:

$$
L=\text { length }\left(D L \_M A P\right)+\text { length }\left(U L \_M A P\right)+3 T_{s}
$$

In addition, we propose that Tsleep is calculated in a way that the sleep interval always ends before Preamble, $\mathrm{FCH}$, and the DL-MAP and UL-MAP messages so that an MSS can send or receive data immediately if there is a sufficient number of available OFDM symbols. The minimum value of Tsleep is calculated as follows:

$$
T_{\text {min }}=\text { FrameDuration }-L
$$

In order to obtain good performance in low traffic load, we propose, as in the existing power saving algorithms, to increment Tsleep without exceeding Tmax. It is up to the operator to choose the value of Tmax. In order to keep listening and sleep windows synchronization, we propose to increment Tsleep as follows:

$$
\text { Tsleep }=\min (\text { Tmax }, N c \times \text { FrameDuration }-L)
$$

where $N c$ represents the number of successive sleep cycles. Note that the extra computations defined in equations (3), (4), and (5) are very simple and therefore they do not add any complexity degree to the behavior of the BS.

\section{SIMULATION RESULTS}

\section{A. Simulation model}

The main parameters of our simulation model including the characteristics of the WiMAX frame and the different power saving algorithms are presented in Table I. Note that the durations of the DL-MAP and UL-MAP messages can vary at each WiMAX frame. In fact, they depend on the number of stations served in the downlink and uplink directions (in the current WiMAX frame), respectively

In this paper, we consider the link adaptation. The Signalto-Noise Ratio (SNR) values are randomly chosen at the beginning of the session. Considering a simple cellular model with three cells per cluster, the SNR distribution can be obtained using classical results of the SNR distribution in cellular networks [20]. Having the SNR values, the Modulation and Coding Scheme (MCS) used is directly deduced. The distribution of the SNRs (and then the associated MCSs) is presented in Table II [21].

The traffic load of the different MSSs is variable as the different users do not have the same QoS requirements. The mean traffic load is assumed to be equal to $100 \mathrm{Kbyte} / \mathrm{s}$. Note that when the traffic load is high, the behavior of all power 
TABLE I

PARAMETERS OF SIMULATION

\begin{tabular}{|c|c|c|}
\hline Designation & Parameters & Values \\
\hline \multirow{5}{*}{ Frame parameters } & DL subframe duration & $6 \mathrm{~ms}$ \\
\hline & UL subframe duration & $4 \mathrm{~ms}$ \\
\hline & OFDM symbol duration & $0.1 \mathrm{~ms}$ \\
\hline & $\mathrm{FCH}$ & $0.2 \mathrm{~ms}$ \\
\hline & Preamble & $0.1 \mathrm{~ms}$ \\
\hline \multirow{3}{*}{ Physical parameters } & Bandwidth $B W$ & $5 \mathrm{MHz}$ \\
\hline & Sampling factor $n$ & $\frac{144}{125}$ \\
\hline & Ratio $G$ & \\
\hline \multirow{2}{*}{$\begin{array}{l}\text { Common Power Saving } \\
\text { parameters }\end{array}$} & $\operatorname{Tmin}(\mathrm{ms})$ & $1,2,4,8$ \\
\hline & $T \max (\mathrm{ms})$ & 64 \\
\hline $\begin{array}{l}\text { PSM, ASMA, and } \\
\text { APSM parameters }\end{array}$ & $L(\mathrm{~ms})$ & {$[0.5,4]$} \\
\hline \multirow[t]{2}{*}{ ASMA parameters } & $A V G$ & $\frac{T \max +T \min }{2}$ \\
\hline & $A V G \max$ & $2 * A V G$ \\
\hline APSM parameter & $T f(\mathrm{~ms})$ & 16 \\
\hline $\begin{array}{l}\text { Sleep mode request and } \\
\text { response messages }\end{array}$ & $\begin{array}{l}\text { MOB_SLP_REQ } \\
\text { MOB_SLP_RSP }\end{array}$ & $0.2 \mathrm{~ms}$ \\
\hline $\begin{array}{l}\text { Burst indication mes- } \\
\text { sage }\end{array}$ & MOB_TRF_IND & $0.2 \mathrm{~ms}$ \\
\hline
\end{tabular}

TABLE II

DISTRIBUTION OF THE MCSS FOR ARRIVING USERS

\begin{tabular}{|c|c|c|c|c|}
\hline $\begin{array}{c}\text { SNR range } \\
(\mathrm{dB})\end{array}$ & Modulation & $\begin{array}{c}\text { Coding } \\
\text { rate }\end{array}$ & $\begin{array}{c}\text { Useful bits } \\
\text { per symbol }\end{array}$ & $\begin{array}{c}\text { Proportion } \\
\text { of users }\end{array}$ \\
\hline \hline$[6,8.5[$ & QPSK & $1 / 2$ & 192 & $30 \%$ \\
\hline$[8.5,11.5[$ & QPSK & $3 / 4$ & 288 & $10 \%$ \\
\hline$[11.5,15[$ & $16-$ QAM & $1 / 2$ & 384 & $20 \%$ \\
\hline$[15,19[$ & $16-$ QAM & $3 / 4$ & 576 & $15 \%$ \\
\hline$[19,21[$ & 64-QAM & $2 / 3$ & 768 & $5 \%$ \\
\hline$[21,+\infty[$ & 64-QAM & $3 / 4$ & 864 & $20 \%$ \\
\hline
\end{tabular}

TABLE III

ENERGY MODEL PARAMETERS

\begin{tabular}{|l|l|}
\hline Station state & Power consumption per second \\
\hline \hline Transmit & $600 \mathrm{mw}$ \\
\hline Receive & $500 \mathrm{mw}$ \\
\hline Idle & $200 \mathrm{mw}$ \\
\hline Sleep & $5 \mathrm{mw}$ \\
\hline
\end{tabular}

saving algorithms will be almost the same as the MSSs have to be always awake. Thus we are interested in low traffic loads.

\section{B. Energy model}

Recall that a station can be either in the Awake or in the Sleep modes. In the Sleep mode, it can be either in the listening or in the sleep window. When being in the sleep window, the station is in a Sleep state: the station cannot receive (or send) any message. The station can receive management messages when being in the listening window. Being in the Awake mode, the station can transmit a frame (data, control or management), receive a frame (data, control or management) or be in the idle state. In the idle state, the station is awake but is neither transmitting nor receiving its bursts. Hence, it makes sense hereafter to consider that a station can be in one of four states: transmit, receive, idle or sleep. Table III gives the energy consumed by a station per second at each one of these four states.

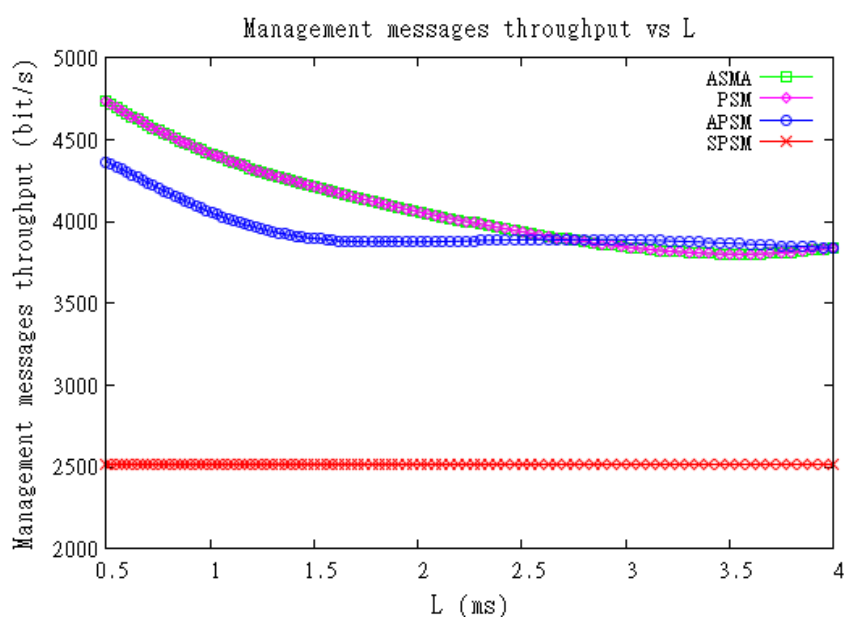

Fig. 5. Management throughput vs listening window $L$.

\section{Investigation of listening window $L$}

In this section, we study how the size of the listening window affects the performance of the power saving algorithms. Note that our proposed algorithm (SPSM) automatically configures this parameter (using equation (3)) while the operator has to choose the adequate value of $L$ when using PSM, ASMA, or APSM.

Figure 5 represents the throughput of the management messages as a function of listening window $L$. We verify that the proposed power saving mechanism provides the lowest management throughput as the synchronization of the listening window allows the BS to gives away the MOB_TRF_IND messages. The management throughput of SPSM is equal to $2500 \mathrm{bit} / \mathrm{s}$ and then it supplies a reduction between $37.5 \%$ and $48 \%$ compared to the existing power saving algorithms.

We also show that when $L$ increases, the management throughput decreases when using PSM, ASMA, or APSM. In fact, the increase of $L$ implies the reduction of the number of sleep cycles in a defined simulation duration. Therefore, the number of the MOB_TRF_IND messages sent is decreased.

Figure 6 represents the mean delivery time as a function of $L$. We observe that SPSM offers the best delivery rapidity to the MSSs. This can be explained by the fact that the BS can deliver data bursts rapidly when the listening and sleep windows are synchronized. In fact, the BS informs and authorizes simultaneously the MSSs to send or receive data. We also remark that PSM and ASMA outperform APSM as this latter power saving algorithm produces more response delay when it tries to achieve higher energy conservation (see Section II-C).

As we can see in Figure 7, SPSM provides the lowest energy consumption compared to other power saving algorithms. This is due to the synchronization and the configuration of different power saving parameters done by our mechanism proposed. This synchronization allows the station to not futilely stay a long time in the wait of the permissions to send and receive data. Therefore, the MSSs stay more time in the Sleep state. 


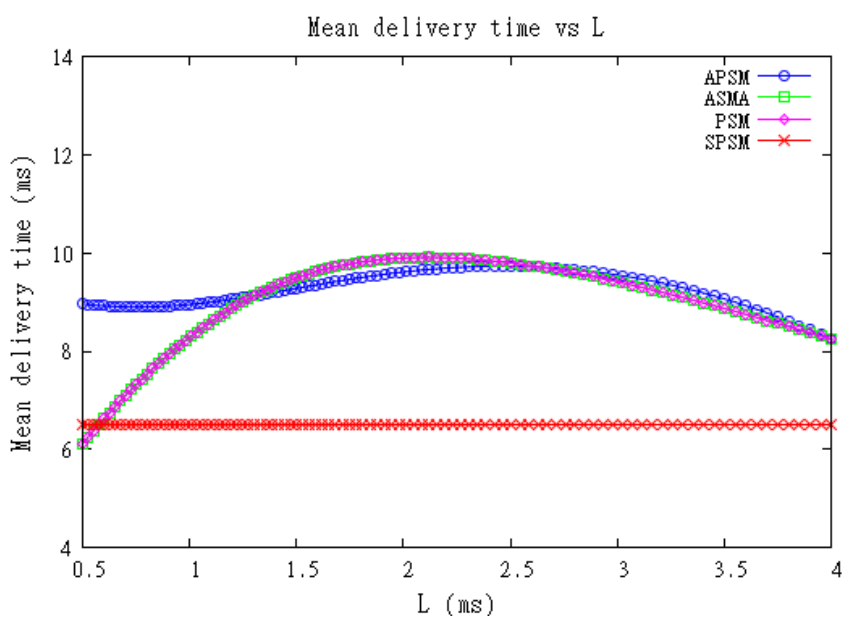

Fig. 6. Mean delivery time vs listening window $L$.

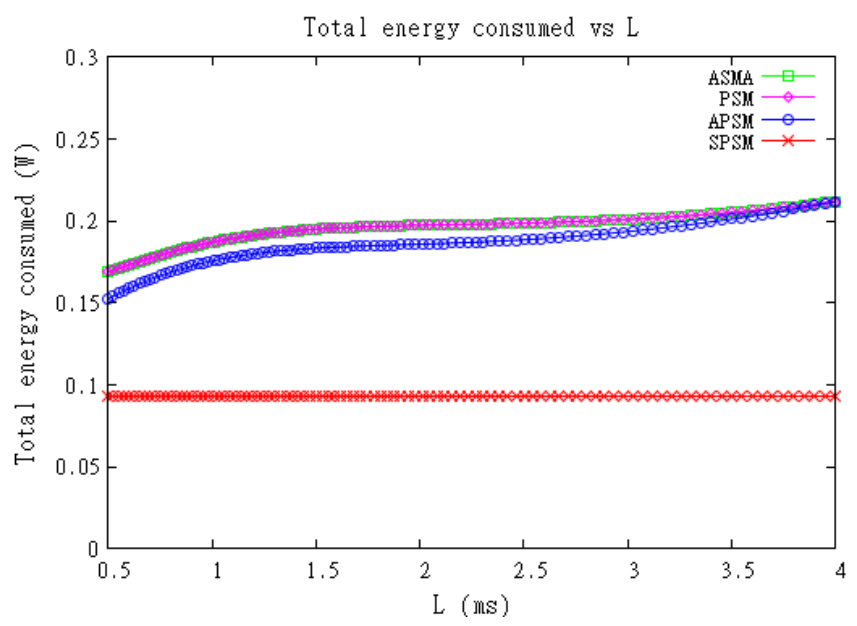

Fig. 7. Total energy consumed vs listening window $L$.

Moreover, as shown in Figure 5, SPSM uses less management messages and so MSSs use less energy for reception. Finally, we verify that APSM outperforms PSM and ASMA in term of total energy consumed.

\section{Investigation of Tmin}

Using equation (4), SPSM automatically configures Tmin while the operator has to choose the adequate value of this power saving parameter when using PSM, ASMA, or APSM.

Figure 8 represents the management messages throughput as a function of Tmin. We verify that SPSM outperforms the existing power saving algorithms by decreasing the management throughput as it shrugs off the MOB_TRF IND messages. For the existing power saving algorithms, the BS uses less MOB_TRF_IND messages when Tmin increases because of the decrease of the number of the sleep cycles. Recall that the BS broadcasts a MOB_TRF_IND message at each sleep cycle.

Figures 9 and 10 represent the mean delivery time and the total energy consumed as a function of Tmin, respectively.

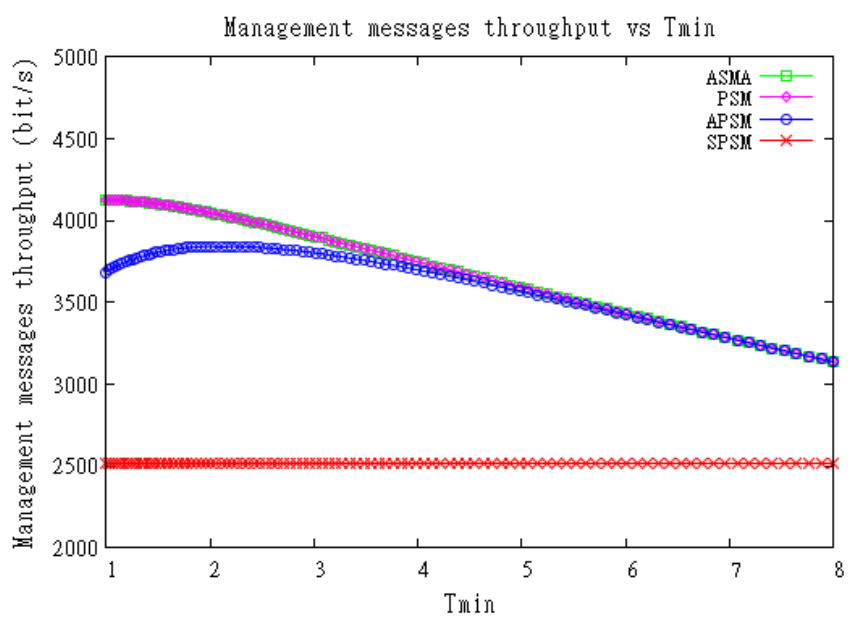

Fig. 8. Management throughput vs Tmin.

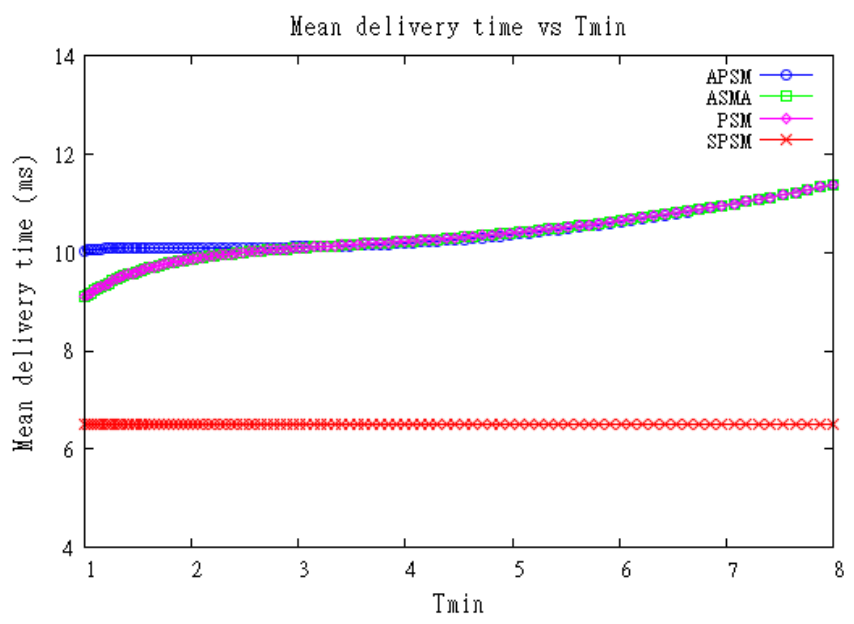

Fig. 9. Mean delivery time vs Tmin.

The simulation results show that our proposed power saving algorithm reduces the delivery delay (between $27.8 \%$ and 43.5\% less) and the energy consumption (between $37.5 \%$ and $52.5 \%$ less). This performance enhancement is achieved due to, on the one hand, the combination between the indication and authorization of burst delivery and, on the other hand, the extension of the sleep window duration when necessary. Finally, we remark that APSM also outperforms PSM and ASMA in term of total energy consumed in spite of the mean delivery time.

\section{CONCLUSion}

In this paper, after briefly presenting the main features of standard IEEE 802.16, we have detailed the principles of the mechanisms of energy conservation proposed for the WiMAX devices. Then, we proposed our enhanced power saving algorithm called SPSM.

Our proposal allows mobile subscriber stations to automatically configure various parameters of energy conservation (especially listening window $L$ and sleep window Tsleep). 


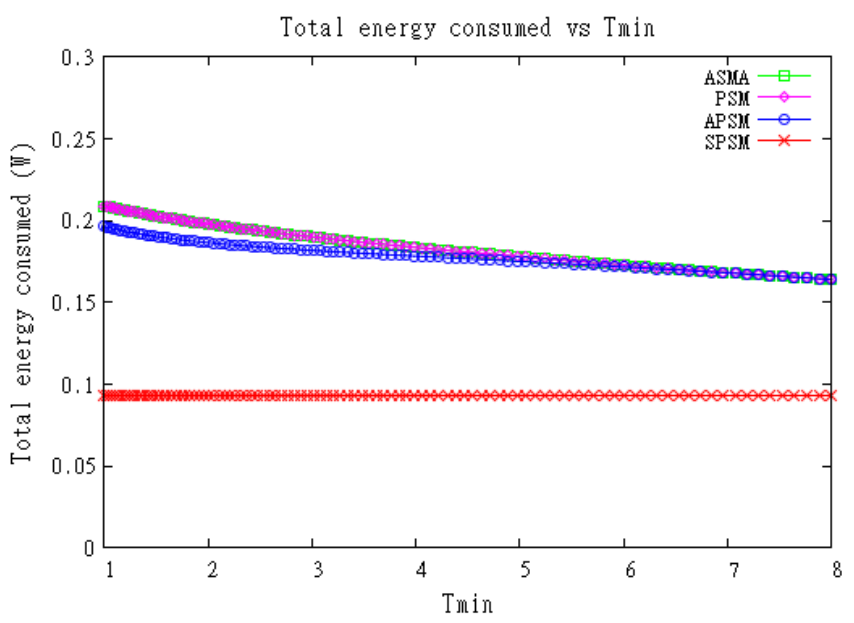

Fig. 10. Total energy consumed vs Tmin.

This automatic configuration is done in a way to wake up the MSS at the best time in order to have an immediate authorization for sending and receiving data. The synchronization and the configuration of the listening and sleep windows allows MSSs, on the one hand, improve the mean delivery time since the station will not have to futilely wait for the next WiMAX frame to send/receive its data. On the other hand, our mechanism will reduce the energy consumption as the MSS remains in the Sleep mode during the time interval between the instants of the indication and authorization deliveries. Moreover, the decrease of the number of the management messages used by the power saving algorithm provides a decrease in the energy consumed

We have shown through extensive simulations that our power saving algorithm (SPSM) outperforms PSM, ASMA, and APSM in terms of mean delivery time, management messages throughput, and total energy consumption. This performance can be improved in future work by taking into account the bandwidth request sent by MSSs whether they use contentions for non-real time services or unicast request opportunities for real time services. Another enhancement can be performed when we consider the battery lifetime for power saving as done in [22].

\section{REFERENCES}

[1] IEEE 802.16-2004, IEEE Standard for local and metropolitan area networks, Air Interface for Fixed Broadband Wireless Access Systems, October 2004.

[2] IEEE 802.16e, IEEE Standard for local and metropolitan area networks, Air Interface for Fixed Broadband Wireless Access Systems, Amendment 2: Physical and Medium Access Control Layers for Combined Fixed and Mobile Operation in Licensed Bands and Corrigendum 1, Feb 2006.

[3] H.J. Thontadharya, D. Shwetha, M. Subramanya Bhat, and J.T. Devaraju, "Effect of Idle Mode on Power Saving in Mobile WiMAX Network", Proceedings of International Conference on Advances in Computing (ICAdC), vol. 174, pp. 491-499, springer India, 2012.

[4] Abdelfettah Belghith, Aymen Belghith, and Miklos Molnar, "Enhancing PSM Efficiencies in Infrastructure 802.11 Networks", Proceedings of International Journal of Computing \& Information Sciences (IJCIS), vol. 5, pp. 13-23, April 2007.
[5] Jia Liu, Chuang Lin, and Fengyuan Ren, "HMM-Based Predictive Power Saving Mechanism in WiMAX", IEEE/ACIS 9th International Conference on Computer and Information Science (ICIS), pp. 459-464, Kaminoyama, Japan, August 2010.

[6] Jia Liu, Fengyuan Ren, and Chuang Lin, "A Dual-Threshold Power Saving Mechanism in WiMAX", IEEE/ACIS 9th International Conference on Computer and Information Science (ICIS), pp. 465-470, Kaminoyama, Japan, August 2010.

[7] Kim Beomjoon, Park Jaesung, and Choi Yong-Hoon, "Power Saving Mechanisms of IEEE 802.16e: Sleep Mode vs. Idle Mode", Frontiers of High Performance Computing and Networking ISPA 2006 Workshops, pp. 332-340, Sorrento, Italy, 4-7 December, 2006.

[8] Shaofei Lu, Jianxin Wang, and Yao Liu, "Modeling and Simulation of Power Saving Mechanism for IEEE 802.16e Mobile WiMAX”, Proceedings of International Journal of Advancements in Computing Technology (IJACT), vol. 3, pp. 418-425, November - December, 2011.

[9] F.M. Abinader, et al., "Performance of Power Saving Modes in IEEE 802.16e System", the 76th IEEE Vehicular Technology Conference (VTC2012-Fall), pp. 1-5, Quebec City, Canada, 3-6 September, 2012.

[10] T. D. Lagkas, P. Sarigiannidis, and M. Louta, "On analyzing the intraframe power saving potentials of the IEEE 802.16e downlink vertical mapping", Proceedings of Computer Networks: The International Journal of Computer and Telecommunications Networking, vol. 57, pp. 16561673, Elsevier North-Holland, May, 2013.

[11] Arun M Bhaskaran, P. Samundeeswari, and M. Harikrishnan, "Adaptive Sleep Mode Mechanism for 3G WiMax Networks", Proceedings of IOSR Journal of Electronics and Communication Engineering (IOSR-JECE), vol. 8, pp. 56-61, November - December, 2013.

[12] C. Mathumathi and T. M. Manjusha, "A QoS-oriented distributed routing protocol for hybrid wireless networks", International Journal of Advanced Research in Computer and Communication Engineering (IJARCCE), vol. 4, Issue 1, January 2015

[13] Woo Jin Jung, Hyung Joo Ki, Tae-Jin Lee, and Min Young Chung, "Adaptive Sleep Mode Algorithm in IEEE 802.16e", Asia-Pacific Conference on Communications (APCC 2007), pp. 483-486, Bangkok, Thailand, October 2007.

[14] Min-Gon Kim, JungYul Choi, and Minho Kang, "Adaptive Power Saving Mechanism Considering the Request Period of Each Initiation of Awakening in the IEEE 802.16e System", IEEE Communications Letters, pp. 106-108, vol. 12, February 2008.

[15] Min-Gon Kim, JungYul Choi, and Minho Kang, "Enhanced PowerSaving Mechanism to Maximize Operational Efciency in IEEE 802.16e Systems", IEEE Transactions on Wireless Communications, pp. 47104719, vol. 8, September 2009 .

[16] Jianbin Xue, Ting Zhang, Songbai Li, and Wenhua Wang, "An adaptive dual-threshold power saving mechanism in WiMAX. Telecommunication Systems", Telecommunication Systems, Volume 53, pp. 131-137, Springer, May 2013.

[17] Ismat Aldmour, "LTE and WiMAX: Comparison and Future Perspective", Communications and Network, vol. 5, pp. 360-368, November 2013

[18] Aymen Belghith and Loutfi Nuaymi, "Scheduling Techniques for WiMAX". In: Maode Ma. "Current Technology Developments of WiMAX Systems", Springer, pp. 61-84, 2009.

[19] Aymen Belghith and Loutfi Nuaymi, "WiMAX capacity estimations and simulation results", IEEE 67th Vehicular Technology Conference, VTC2008-Spring, pp. 1741-1745, Marina Bay, Singapore, 11-14 May 2008.

[20] Xavier Lagrange, "CIR cumulative distribution in a regular cellular network", Technical report, TELECOM Bretagne, 2000.

[21] Aymen Belghith, Loutfi Nuaymi, and Patrick Maillé, "Pricing of RealTime Applications in WiMAX Systems", IEEE 68th Vehicular Technology Conference, VTC2008-Fall, Calgary, Canada, 21-24 September 2008.

[22] Ibrahim Saidu et al., "An Efficient Battery Lifetime Aware Power Saving (EBLAPS) Mechanism in IEEE 802.16e Networks"', Wireless Personal Communications, Springer, vol. 80, Issue 1, pp. 29-49, January 2015, 